Maciej K. Bieliński ${ }^{1,2}$, Aleksandra Chyrek-Tomaszewska², Alicja Popiołek ${ }^{1,2}$, Agnieszka Stachowicz-Karpińska², Marta Dura ${ }^{3}$, Grzegorz Pulkowski²

${ }^{1}$ Department of Clinical Neuropsychology, Faculty of Health Sciences, Nicolaus Copernicus University in Toruń, Collgium Medicum

in Bydgoszcz, Poland

2Clinic of Vascular and Internal Diseases, Jan Biziel's University Hospital No. 2, Bydgoszcz, Poland

${ }^{3}$ Department of Radiology, Jan Biziel's University Hospital No 2, Bydgoszcz, Poland

\title{
Giant heart tumor - hope not all lost
}

A 28-year-old man was admitted to the hospital due to strongly expressed dyspnoea, cough and peripheral oedema. The above symptoms started about 4 weeks before the admission and had significantly increased in the last 2 days. In addition, he reported the occurrence of papulopustular, itchy rash for several months. In 2013 and 2014, the patient underwent chemotherapy and radiotherapy for Hodgkin's lymphoma (HL) which resulted in obtaining a remission. No other medical history was presented. At the admission, for a screening test, a transthoracic echocardiography was made which revealed a large amount of pericardial fluid and pathological mass in the heart. A pericardiocentesis was performed promptly to decompress the cardiac tamponade and to collect material for histopathological examination. Unfortunately, immunohistochemistry did not allow to make a diagnosis. After the pericardial drainage, the patient's condition was stabilized, enabling further diagnostic processes. Initially, we decided to perform a full echocardiographic examination, that showed irregular, heterogeneous masses which were encompassing the base of the heart, filling the right ventricle and the majority of the right atrium. Subsequently, we utilized imaging studies to determine the size of the lesion and the extent of its infiltration. Computed tomography scan and magnetic resonance imaging of the chest revealed the presence of fluid in pleural cavities and pericar- dial sac, as well as the distorted image of the heart due to advanced infiltration. The pathological mass was extending from upward and downward of the cardiac wall, penetrating the interventricular septum, and growing into the right heart cavities. The lesion adhered tightly to the anterior chest wall, causing the mass effect on the left atrium. Moreover, the tumour was covering and pressing on big vessels, such as lung trunk, the aorta, left carotid artery and subclavian artery. In addition, numerous enlarged lymph nodes were observed in the mediastinum. Subclavian and cervical lymph nodes were palpable during the physical examination. Regarding the history of $\mathrm{HL}$, the lymph node biopsies were evaluated in the histopathological examination, which described findings typical to HL. Immediate standard treatment was started with adriamycin, bleomycin, vinblastine and dacarbazine. PET-CT scan, conducted after two treatment cycles, revealed numerous, enlarged, diseased lymph nodes, and the patient had been qualified for chemotherapy with brentuximab vedotin and bendamustin. After two treatment cycles, the PET-CT study was repeated. The image of nodal lesions corresponded to the significant metabolic and morphological regression, and significant reduction of cardiac lesions was described. The patient's condition has normalized, all symptoms have disappeared and the physical capacity has returned. The patient continues haematological treatment.

\section{Corresponding author:}

Maciej K. Bieliński, Jan Biziel's University Hospital No. 2, Clinic of Vascular and Internal Diseases,

Kornel's Ujejski 75 St. 85-168 Bydgoszcz, Poland, e-mail: bielinskim@gmail.com

Medical Research Journal 2018;

Volume 3, Number 3, 179-180

10.5603/MRJ.2018.a0023

Copyright (C) 2018 Via Medica

ISSN 2451-2591 

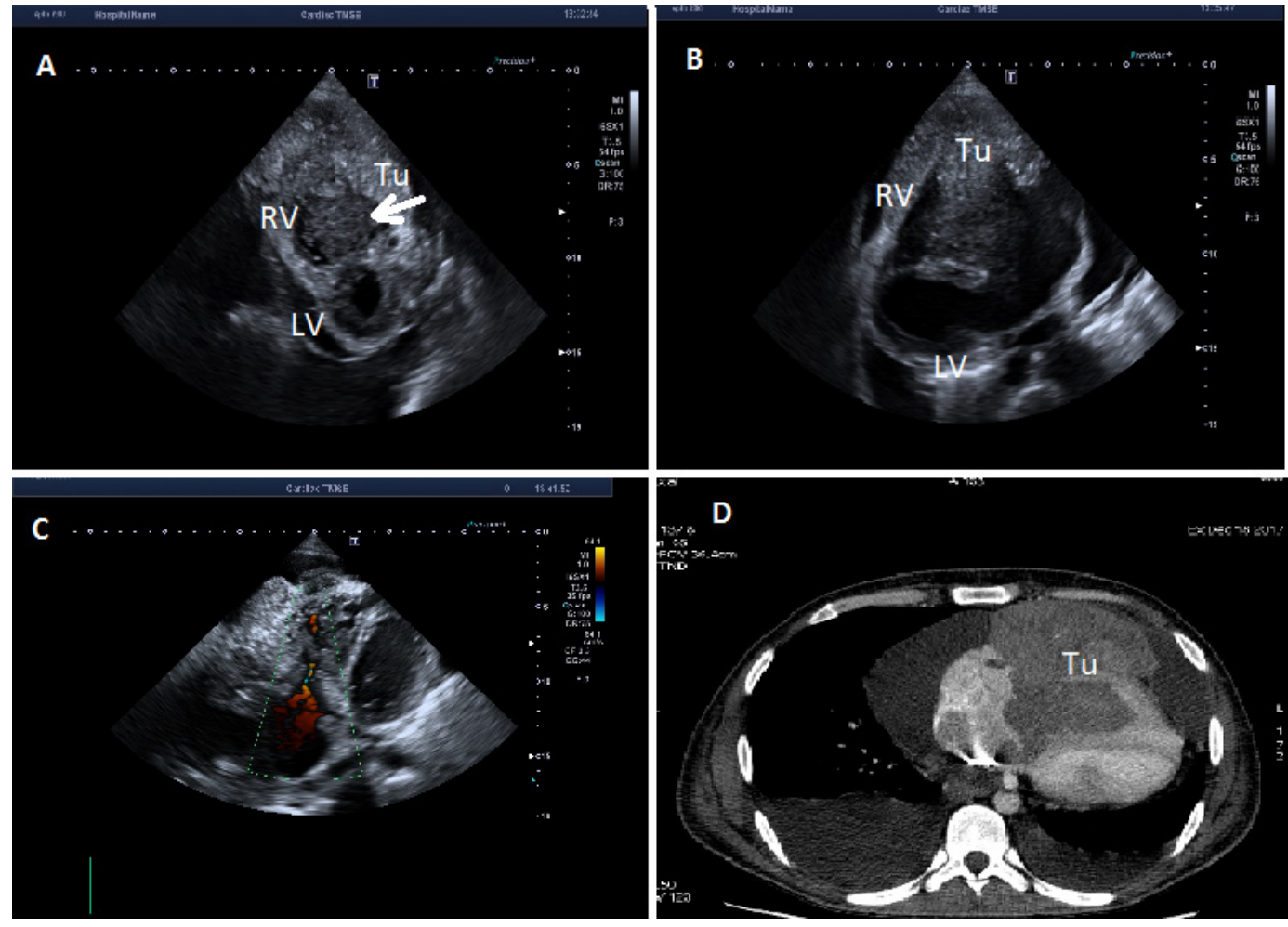

Figure 1. Legend

A - Echocardiography - parasternal short axis projection - pathological mass filling the right ventricle

B - Echocardiography - parasternal long axis projection - pathological mass filling the right ventricle

C - Echocardiography - untypical apical projection - preserved residual flow through the right ventricle

D - Magnetic resonance - pathological mass filling the right ventricle, passing through the free wall of the right ventricle, reaching to the wall of the chest

RV - right ventricle

LV - left ventricle

Tu - tumour

\section{Discussion}

$\mathrm{HL}$ metastases in the heart are described on the basis of sectional studies in approximately $25 \%$ of cases (1). Occasionally, the cardiac manifestation of $\mathrm{HL}$ in the form of arrhythmias, heart failure, cardiac tamponade, overtakes other symptoms (2). It should be noted, that the pericardial fluid does not have to result from $\mathrm{HL}$ metastases to the heart tissues. It may be associated with impaired flow caused by the blockage of lymphatic or/and venous drainage of pericardial fluid associated with neoplasmatic cells or the compression of enlarged lymph nodes (3). The existence of cardiac alterations in the course of HL may hinder the evaluation of late effects of treatment. Therefore, the prognosis should be determined on the basis of typical criteria (4).

\section{Conflict of interest: none declared}

\section{References}

1. Roberts W, Glancy D, Devita V. Heart in malignant lymphoma (Hodgkin's disease, lymphosarcoma, reticulum cell sarcoma and mycosis fungoides). The Am J Cardiol. 1968; 22(1): 85-107, doi: 10.1016/00029149(68)90250-6.

2. Amirimoghaddam Z, Khoddami M, Nayeri ND, et al. Hodgkin's lymphoma presenting with heart failure: a case report. J Med Case Rep. 2010; 4: 14, doi: 10.1186/1752-1947-4-14, indexed in Pubmed: 20205760.

3. Hajra A, Bandyopadhyay D, Layek M, et al. Cardiac Tamponade as Initial Presentation of Hodgkin Lymphoma. J Clin Imaging Sci. 2015; 5: 67, doi: 10.4103/2156-7514.172971, indexed in Pubmed: 26900491.

4. Non-Hodgkin's Lymphoma Clinical Practice Guidelines in Oncology. J Natl Comp Canc Netw. 2004; 2(4): 286-286, doi: 10.6004/jncen.2004.0027. 